\title{
هدى تطبيق عناصر العملية الإدارية على الإدارة المنزلية
}

\author{
إعــــــاد \\ د/ أميره احمد سالم بالخيور \\ أستاذ مساعد السكن وإدارة المنزل \\ بكلية التربية للاقتصاد المنزلي - جامعة أم القرى
}

مجلة بحوث التربية النوعية - جامعة المنصورة

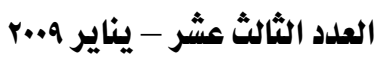




\section{مدى تطبيق عناصر العملية الإدارية على الإدارة المنزلية}

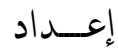

د/أمبرهاحمد سالمبالجيور

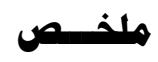

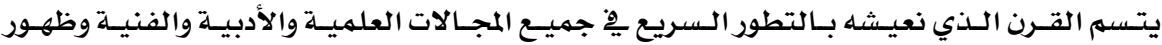

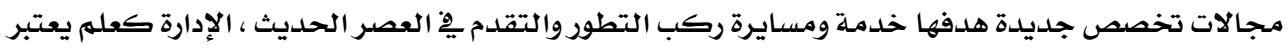

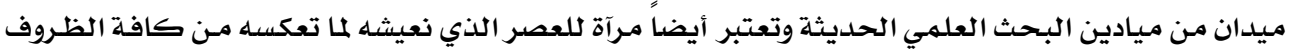

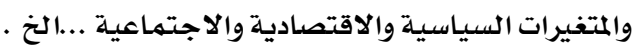

ولكي تتمكن الإدارة من انجاز المسؤوليات وتحقيق الأهداف بأقصى درجة من الكفاءة لابد أن تؤخذ بِّة

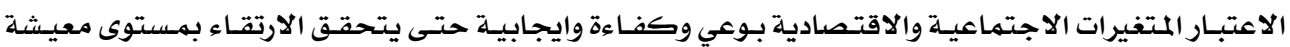

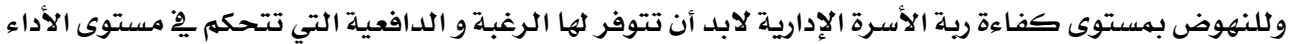

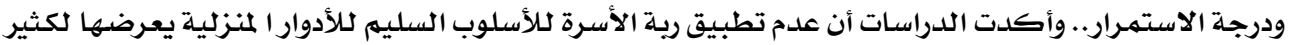

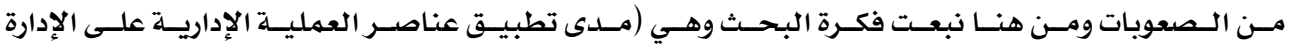

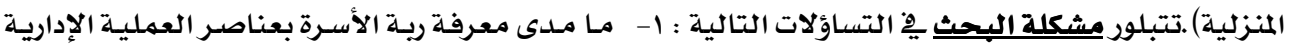

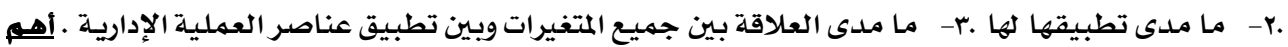

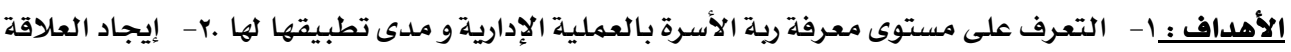

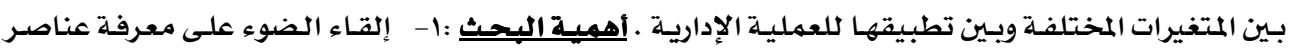

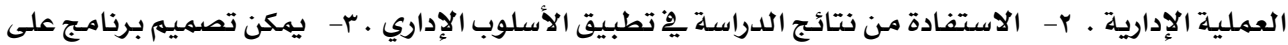

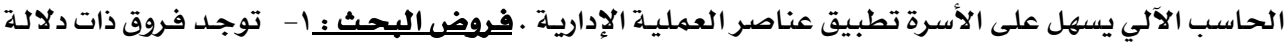

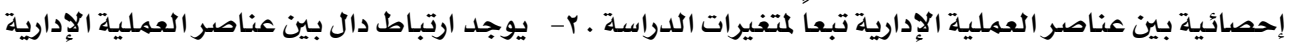

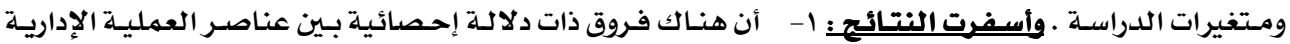

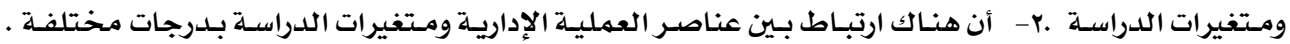

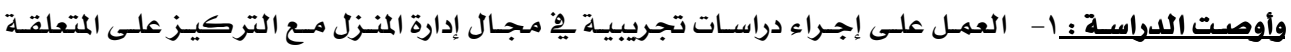

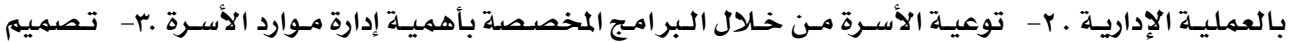

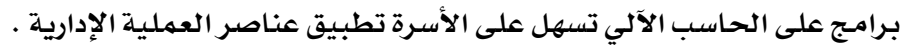




\section{ABSTRACT}

\section{Implementation Scope Of Management Process Upon Home Management}

We live in a century that is known to be developing fast in all fields of science, literature and arts. As well as the appearance of some new fields of expertise that aim to serve and accompany development and advancement in the new age. Management as a science, is considered to be a field of modern scientific research, as well as a mirror for the age we live in, as for what it reflects of political, economical and social variables and circumstances.

In order for management to accomplish responsibilities and goals meant, with the highest levels of sufficiency, we must carefully, sufficiently, and positively consider economical and social variables to achieve highness in our life scale. Moreover, to raise the sufficiency management level of housewives, she must acquire desire and motivation, which control performance and continuity measurement. Studies evidences indicate that non-application of the right style of house roles faces her with multi difficulties. We here come with the suggestion of research (Implementation Scope of Management Process Upon Home Management).

The research conflict is crystallizes on the following inquiries; How much do housewives know of the Management Process Elements? And how much they apply them? what is the relationship between all variables and implication of the management process elements?

objectives: 1) To Identify the housewife's knowledge with the management process, and scope of application. 2) Recognizing the relationship between different variables and their applications upon the management process.

Importance of Paper: 1) Spotlighting Elements of the Management process. 2) Benefiting from results in applying the managerial style. 3) Generalizing a computer program to facilitate the application of management process elements by the family.

Prescriptions of Paper: 1) There are a Statistic Indicating Prescriptions among the elements of management process according to the study variables. 2) 


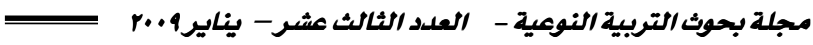

The existence of an indicating link between the management process elements and the study variables.

Results indicated that; 1) There are statistic indicating prescriptions between the elements of management process and the study variables. 2) A link between the managerial process elements and the study variables in different scales.

The study however recommended; 1) To conduct experimental studies in home management, focusing on the relationship with management process. 2) And to increase aware family through specialized programs to the importance of family resources. 3) Generalization of a computer program which facilitates application of management process elements by the family. 
يتسهم القرن الذي نعيشه بالتطور السريع ٌِِ جميع المجالات العلمية والأدبية والفنية وظهور

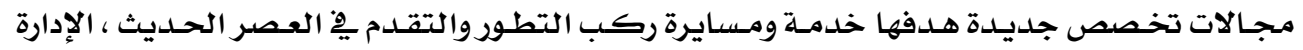

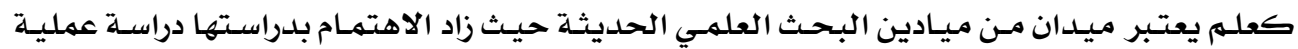

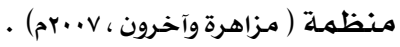

وتعد الإدارة مرآة للعصر الذي نعيث فيه لما تعكسه مـن كافـة الظروف والمتغيرات السياسية



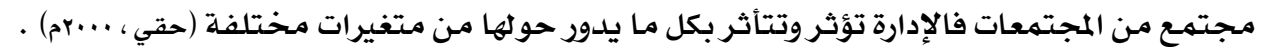

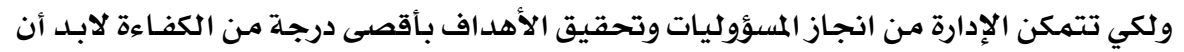

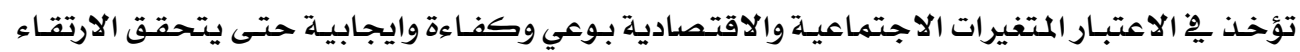
بمستوى معيشة (حقي ، ... بوم) .

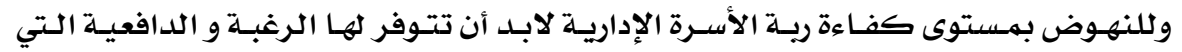

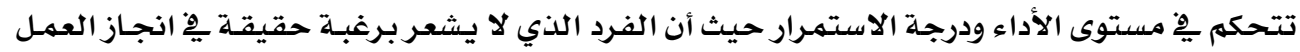

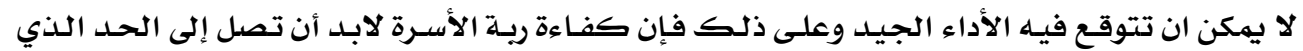

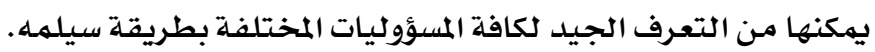

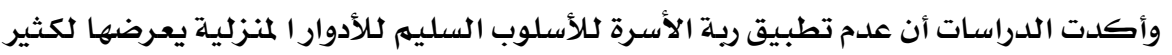

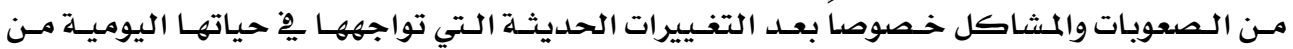

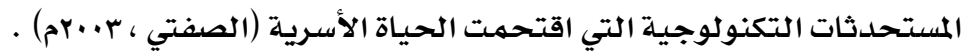

ومن هنا نبعت فكرة البحث وهي ( مدىى تطبيق عناصر العملية الإدارية على الإدارة المنزلية )

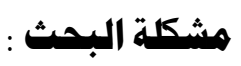

$$
\text { تتبلور مشكلة البحث يِّ التساؤلات التالية : }
$$

ا- ما مدى معرفة رية الأسرة بعناصر العملية الإدارية .

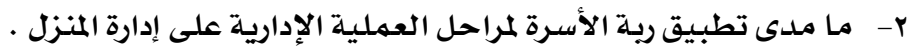

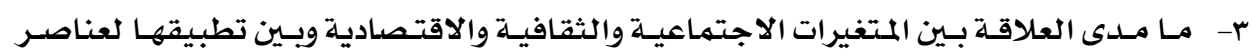

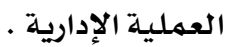

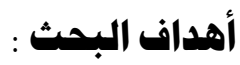

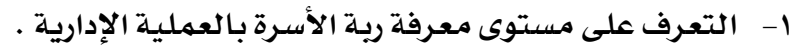

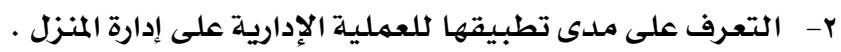

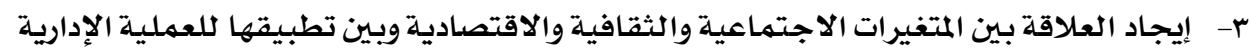




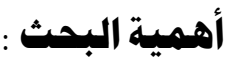

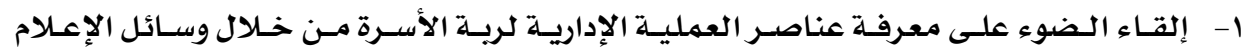
المختلفة .

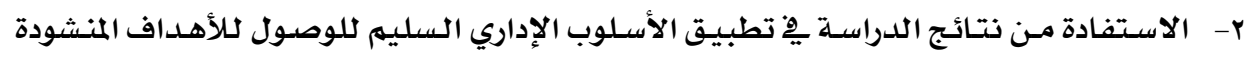

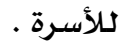

r- يمكن تصميم برنامـج على الحاسب الآلي يسهل على الأسرة تطبيق عناصر العملية الإدارية .

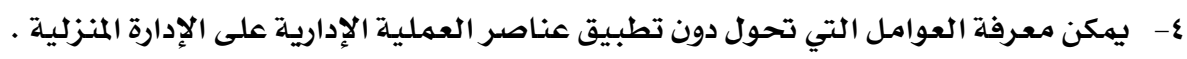

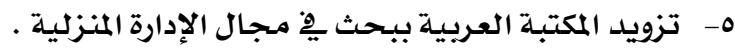

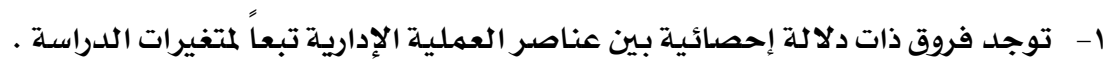

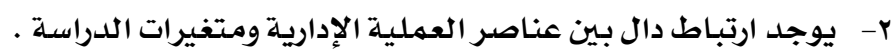

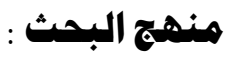

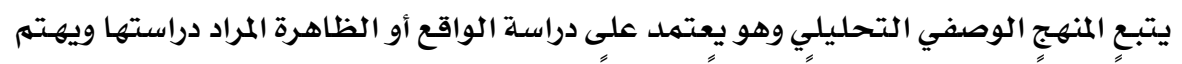

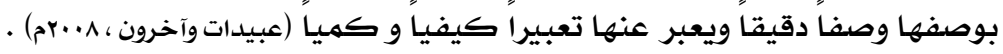

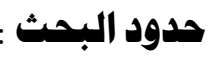

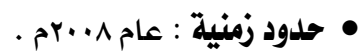

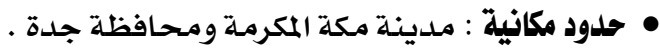

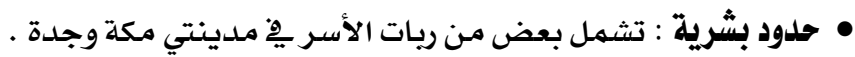

أدوات البحث تحود

ا- استمارة استبيان · الحاسب الآلي لاستخراج العمليات الإحصائية .

الإطار النظري :

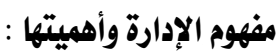

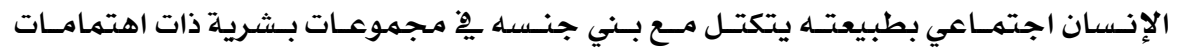

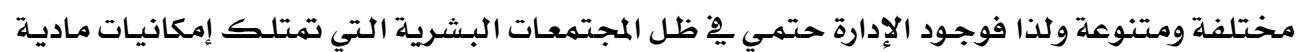

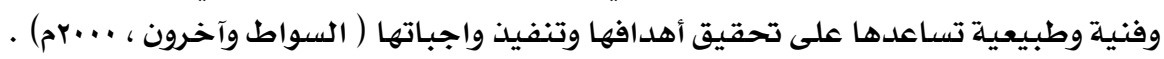

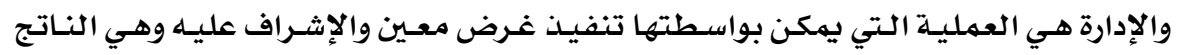

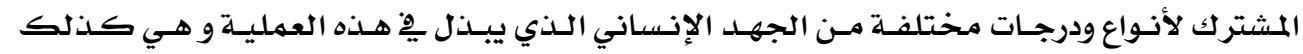

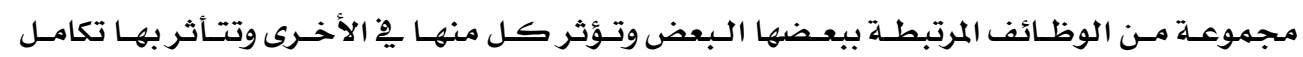

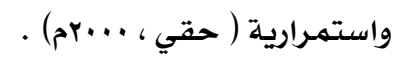

والإدارة العامـة تتضمن أريع عناصر هي : 
ا- التخطيط : هـو إقـرار بـأن يجـب أن يتجـه التتظيم وكيفيـة الوصـول إلى تلك الوجهـة ، وهـذا

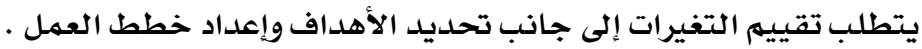

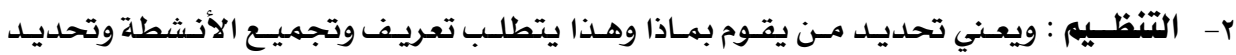

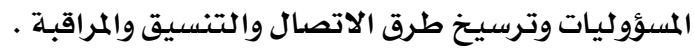

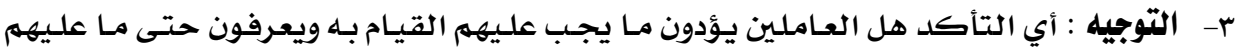

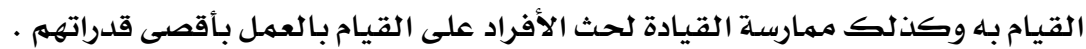

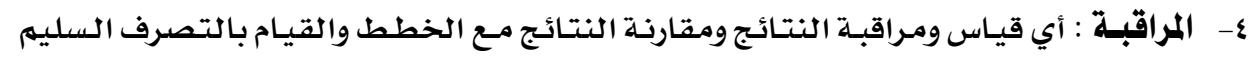
عند الحاجة (ارمسترونج، ؟..بrم).

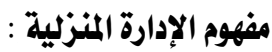

تعـني الإدارة المنزليـة بـالدرجــة الأولى إدارة شـؤون الأسـرة واسـتعمال مـا لـديها مـن إمكانيـات

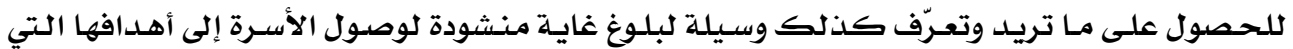
تستغل لصالح أفرادها ( مزاهرة وآخرون ، ... ب). أهداف دراسة الإدارة المنزلية : ا- تكوين اتجـاه التفكير العلمسي السليهم ِِّ اتخـاذ القـرارات ِِّ جميـع مواقف الحيـاة الشخصية والأسرية . بوين التحاه

r- ت تنمية الوعي التخطيطي وتطبيق العملية الإدارية بمراحلها المختلفة يِّ حل المشكلات .

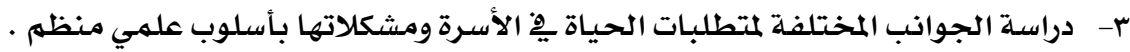

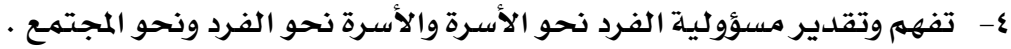

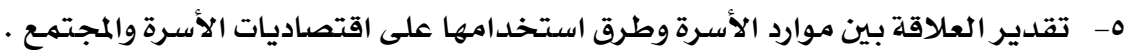

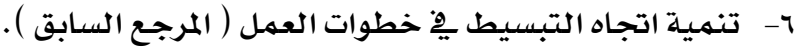
عناصر العمبلية الإدارية : أولاًا : هفهوم التخطيط التهمئ وخصائصه :

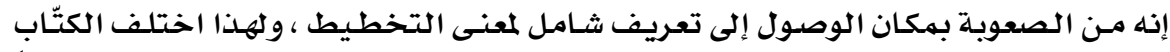

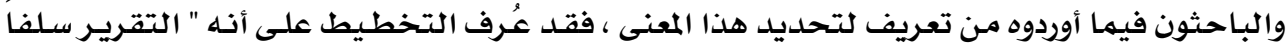

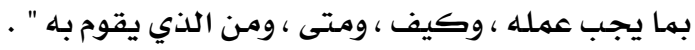

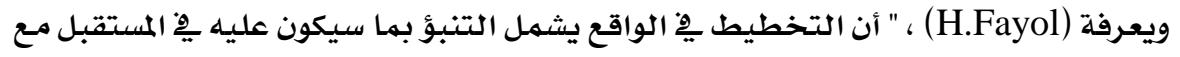
الاستعداد لهذا المستقبل " (توفيق، 1919 م) . ويتصف التخطيط بثلاث خصائص تعد بمثابة ثلاثة تحديات للإدارة المعنية وهي : 1- توقعي ومستقبلي 2ِ طبيعته :

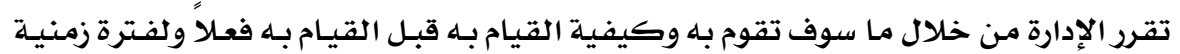


r- يترجم بجملة من القرارات والإجراءات :

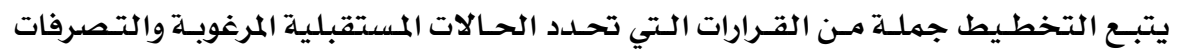

المطلوبة لإنجازها المان.

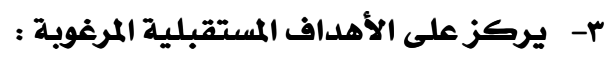

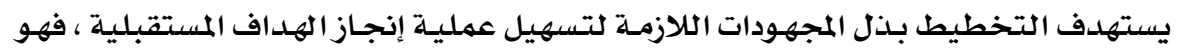

بمثابة الوسيلة و الإدارة لضمان تحقيق هذه الأهداف (الخشروم ، مصطفى ، 1991م) .

مقومات التخطيط : بونابه الوسيله

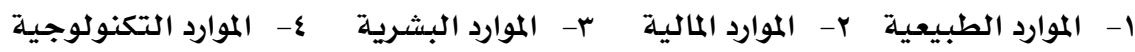

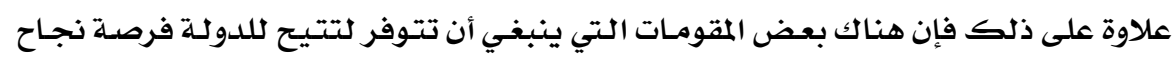

التخطيط ومنها :

ا- توافر المناخ العام الذي يتقبل التخطيط ولا يعارضده .

r- وجود مخططين يتملكون مهارات عقلية وفكرية وإنسانية وفنية ترتبط بالتخطيط .

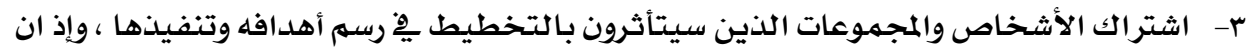

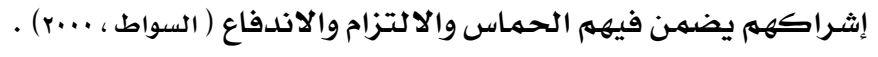

r



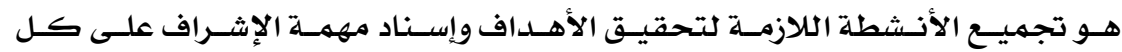

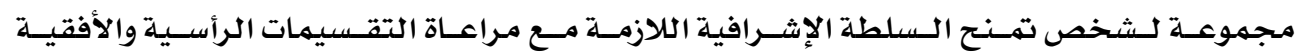

(Starling,1982)

\section{ثالثًا : القيادة والتهوجيه :}

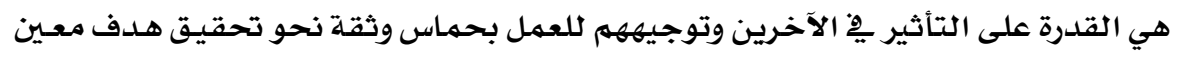

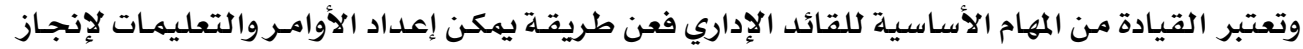

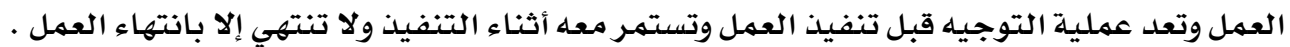

\section{رابعًا : التقيييم والرقابة : التوجية}

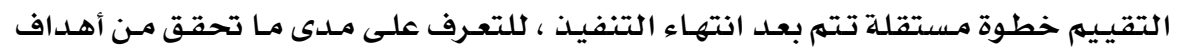

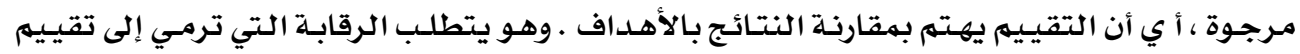

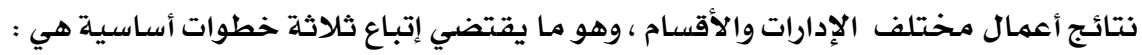




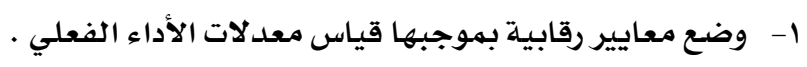

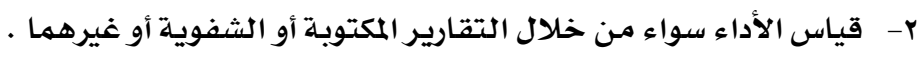

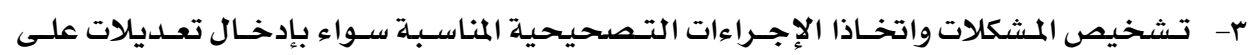

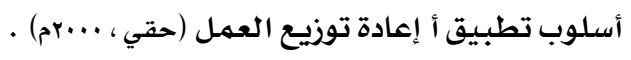

أهمية التقيييه في إدارة المنزل :

$$
\begin{aligned}
& \text { 1- تحسين نوعية ومستوى الأهداف . }
\end{aligned}
$$

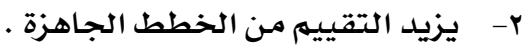

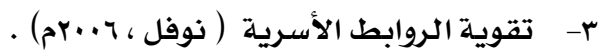

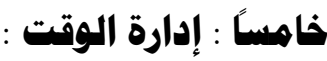

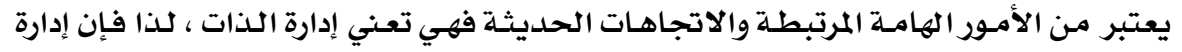

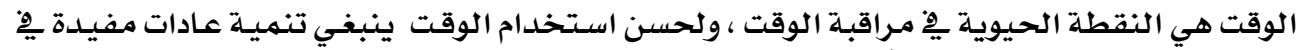

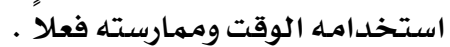

إن مفهـوم إدارة الوقت " عمليـة مستمدة مـن التخطـيط والتحليـل والتقيسيه المستمر لكسل

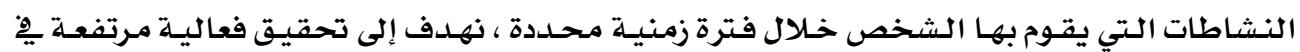

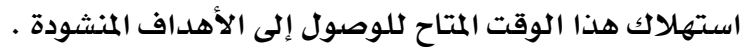

خصائص الوقت :

للوقت خصائص يتميز بها ومنها :

1- سرعة إنقضائة فهو يمر مـر السحاب ، ومثلما تذهب الساعة الأولى من اليوم يذهب اليوم كله r- ما مضى منـه لا يعود مرة أخرى ولا يمكن تجزئتته .

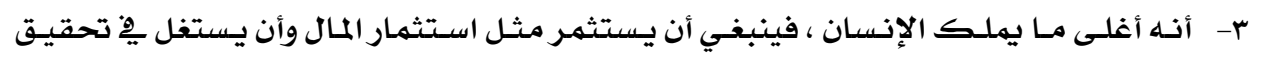

الأهداف .

ع- أنه مورد يتساوى فيه الجميع فالكل لدية ؟ج ساعة يومياً ( زيتون ، بدون ).

المحلددات المؤثرة في أسلوب إدارة الأسرة لمواردها :

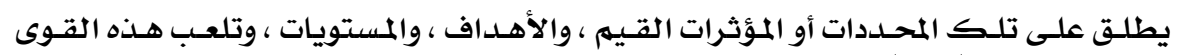

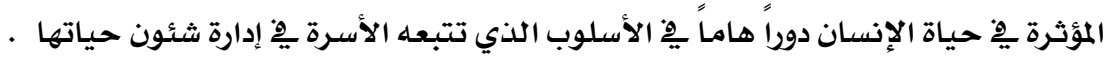

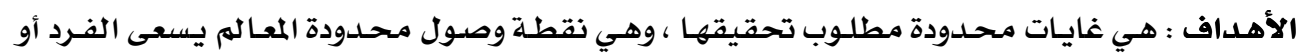

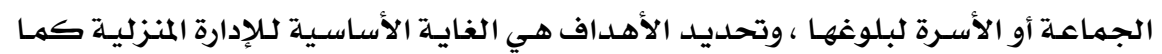

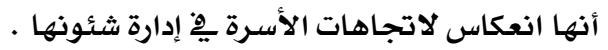

\section{r...}




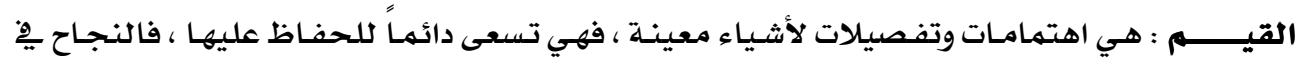

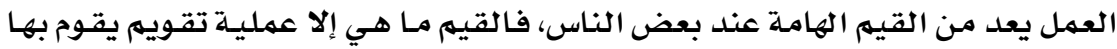

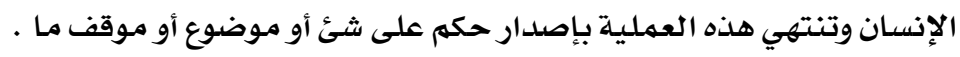

المستويات : تعبر كلمة مستوى بصفة عامـة عن درجة من الكمال يرتضيها الفرد ويعتبرها ضـرورية .

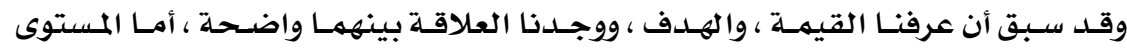

فيصعب تحديد علاقته بأي منهما .

\section{الدور المتكاهل لإدارة المنزل في حياة الفرد والأسرة :}

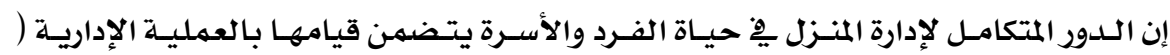

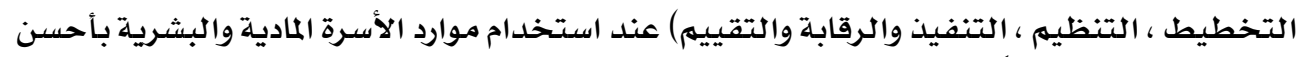

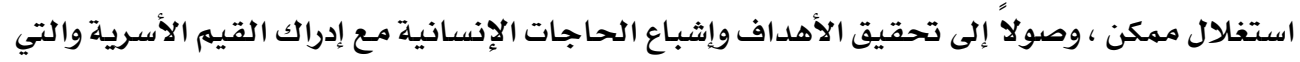
تعد من الدوافع الأساسية وراء تحقيق إلى تلكيق الأهد الأهداف.

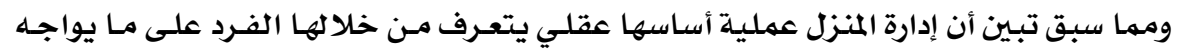

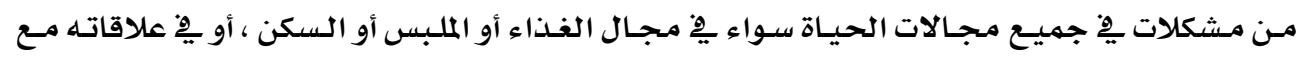

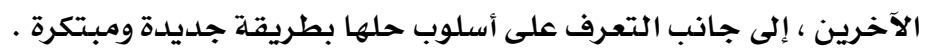

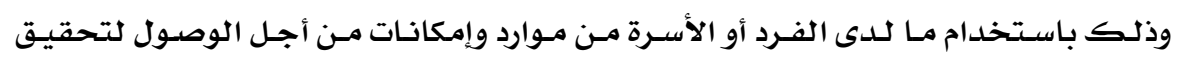

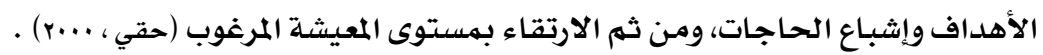

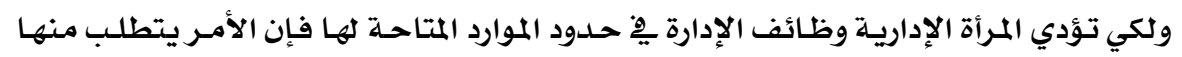
القيام بعدة أدوار :

$$
\begin{aligned}
& \text { 1- تحمل المسؤولية . }
\end{aligned}
$$

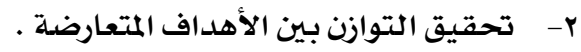

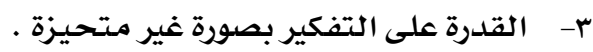

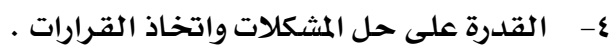

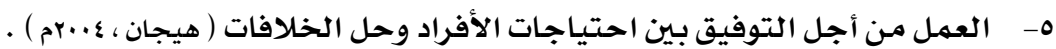

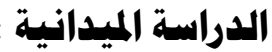 الصداق والثبات :

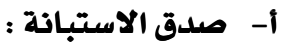

تم حسـاب الصدق باستخلدام الاتسـاق الداخل بين كل من عناصر العملية الإداريـة والجدول

$$
\text { (1) يوضتح ذلك حساب }
$$




\begin{tabular}{|c|c|c|}
\hline الدلالة & معامل الارتبـاط & \\
\hline$\cdot, \cdot 1$ & $\cdot, 9 \cdot v$ & التخطيط \\
\hline$\cdot, \cdot 1$ & $\cdot, \wedge \mathrm{V}$. & التتفيذ \\
\hline$\cdot, \cdot 1$ & - vor & التقييم \\
\hline$\cdot, \cdot 1$ & $\cdot, \wedge \varepsilon$. & إدارة الوقت \\
\hline
\end{tabular}

ب- تم حساب الثبات باستخدام معامل الفا - التجزئة النصفيـة والجدول (r) يوضـح ذلك . جدول (r ) يوضسح الثبات

\begin{tabular}{|c|c|c|}
\hline التجزئة النصفية & معامل ألفا & \\
\hline$\cdot, \Lambda V \Psi-\cdot, \mathrm{VV} \varepsilon$ & $\cdot, \Lambda \cdot \varepsilon$ & التخطيط \\
\hline$\cdot, ৭ \& \cdot-\cdot, \wedge \wedge т$ & $\cdot, \wedge \circ Y$ & التتفيذ \\
\hline$\cdot .989-., 909$ & $\cdot, 9 Y 1$ & التقييم \\
\hline$\cdot, 917-\cdot, \wedge \leqslant 0$ & $\cdot, \mathrm{V} 9 \mathrm{~V}$ & إدارة الوقت \\
\hline$\cdot, q \cdot 1-\cdot, \Lambda Y I$ & $\cdot, \wedge 9 \varepsilon$ & الاستمارة كلها \\
\hline
\end{tabular}

جميع هذه القيهم دال عند أ.

\section{نتائج الدراسة الميدانية :}

قامت البـاحثة بالدراسـة الميدانية لتحقيق أهداف الدراسلة والتي استحدثت على :

$$
\begin{aligned}
& \text { أ- دراسـة العينـة من حيث المستوى التعليهي - المهنـة - حجم الأسـرة . } \\
& \text { ب- معرفة الارتباط بين عناصر العملية الإدارية ومتغيرات الدراسلة . . }
\end{aligned}
$$

\begin{tabular}{|c|c|c|}
\hline النسبـة & العدد & المستوى التعليهي \\
\hline$\%$ IV, $\varepsilon$ & rq & اقل مـن مـتوسط \\
\hline$\% r \cdot, q$ & ro & متوسط \\
\hline$\%$ \%ะ, 1 & ov & ثانوي \\
\hline$\% Y V, 0$ & $\varepsilon 7$ & جامعي فأعلى \\
\hline$\% 1 \ldots$ & 178 & المجموع \\
\hline
\end{tabular}

أولاً : دراسة العينة : 


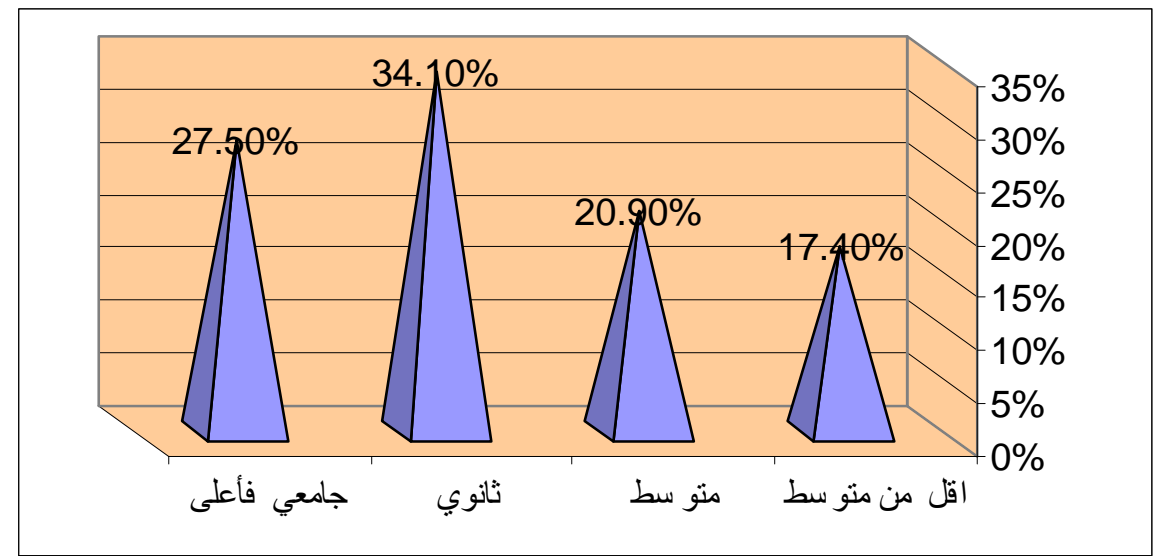

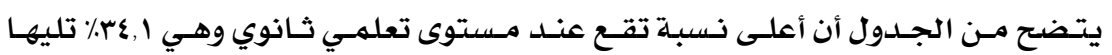

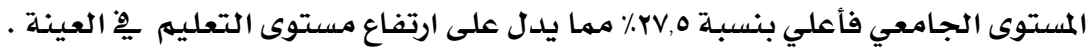
جدول ( ع ) يوضـح المهنة

\begin{tabular}{|c|c|c|}
\hline النسبـة & العدد & المهنة \\
\hline$\% 00, \mathrm{~V}$ & q & ربـة منـزل \\
\hline$\% \varepsilon \varepsilon, r$ & $V \varepsilon$ & موظفة \\
\hline$\% 1 \ldots$ & $17 \mathrm{~V}$ & المجموع \\
\hline
\end{tabular}

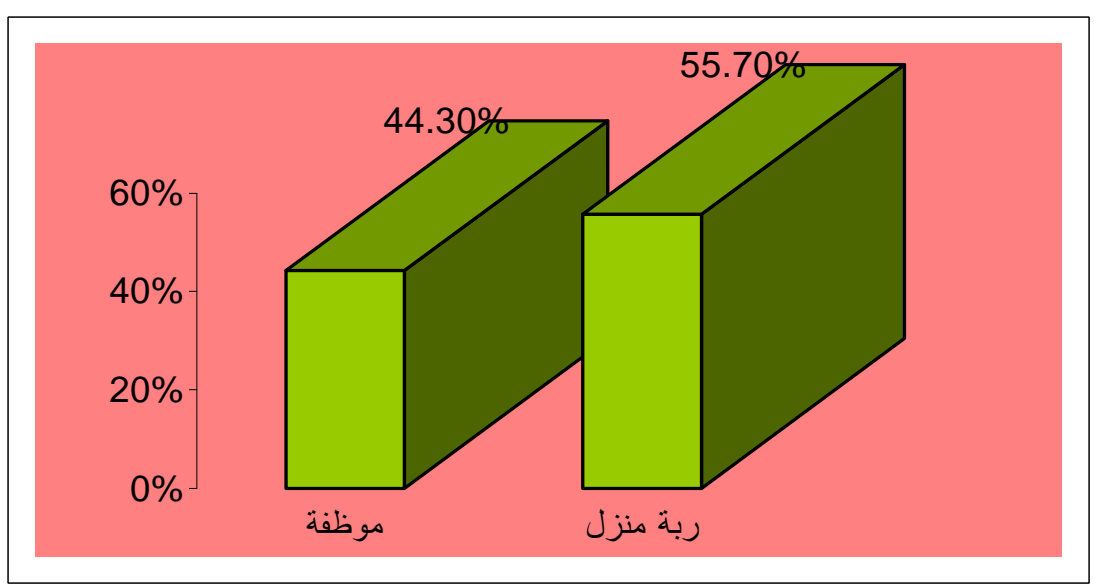

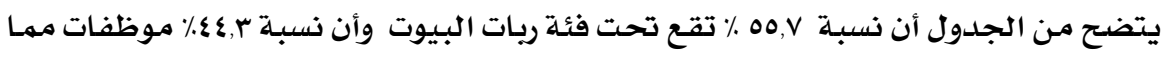

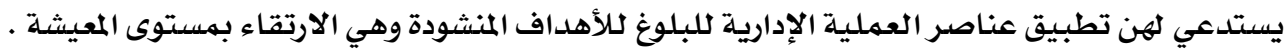


جدول ( ه يوضح حجم الأسرة(عدد الأبناء)

\begin{tabular}{|c|c|c|}
\hline النسبة & العدد & حجم الأسـرة(عدد الأبناء) \\
\hline$\%$ \% , 0 & 77 & واحد إلى ثلاث \\
\hline$\% r V, 1$ & $\pi$ & أربـع إلى ست \\
\hline$\%$ \%,$\varepsilon$ & rq & أكثر مـن ست \\
\hline$\% 1 \ldots$ & $17 \mathrm{~V}$ & المجموع \\
\hline
\end{tabular}

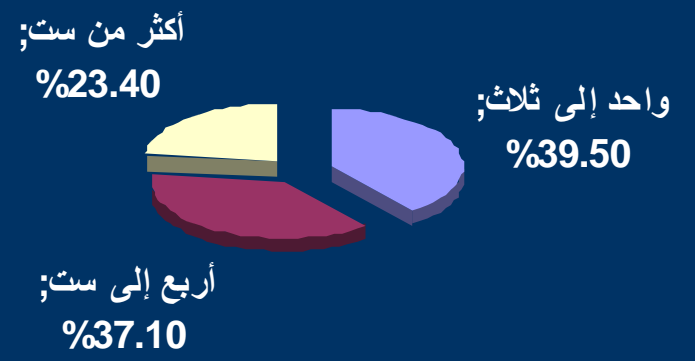

\%37.10

شكل ( 7) يوضح حجم الأسرة(عدد الأبناء)

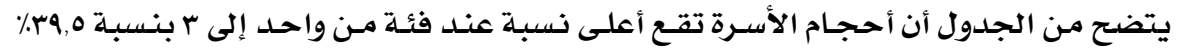

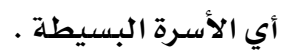

جدول ( ج) يوضح عدد الخدم هِّ الأسرة

\begin{tabular}{|c|c|c|}
\hline النسبـة & العددد & عدد الخدم ِِِ الأسـرة \\
\hline$\%$ \% , V & $\Lambda \Lambda$ & واحد إلى اثنان \\
\hline$\% \varepsilon \vee, r$ & Vq & أكثر مـن اثنان \\
\hline$\% 1 \ldots$ & $17 V$ & المجموع \\
\hline
\end{tabular}




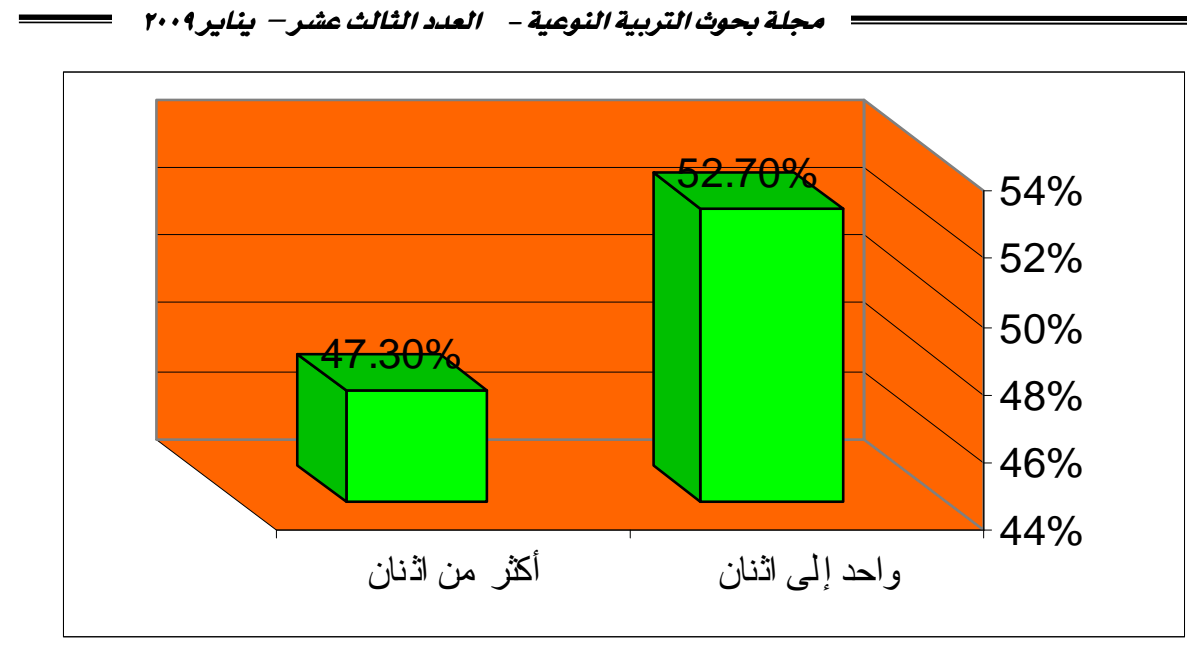

$$
\text { شكل ( ع) يوضح عدد الخدم هِي الأسرة }
$$

يتضح من الجدول أن جميع الأسـر لـديها خـدم ويتفـاوت عدد الخـدم وإنما أعلى نسبة وهي الخدي

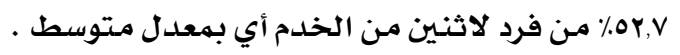

\begin{tabular}{|c|c|c|}
\hline النسبـة & العدد & السن \\
\hline$\%, Y, Y$ & $r v$ & مـن ro ro-r \\
\hline$\%$ \% r,r & $\varepsilon \varepsilon$ & 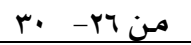 \\
\hline$\%$ \% , I & $\varepsilon V$ & من اس- مر \\
\hline \%,rr, & rq & من بس فأكثر \\
\hline$\% 1 \ldots$ & $17 \mathrm{~V}$ & المجموع \\
\hline
\end{tabular}

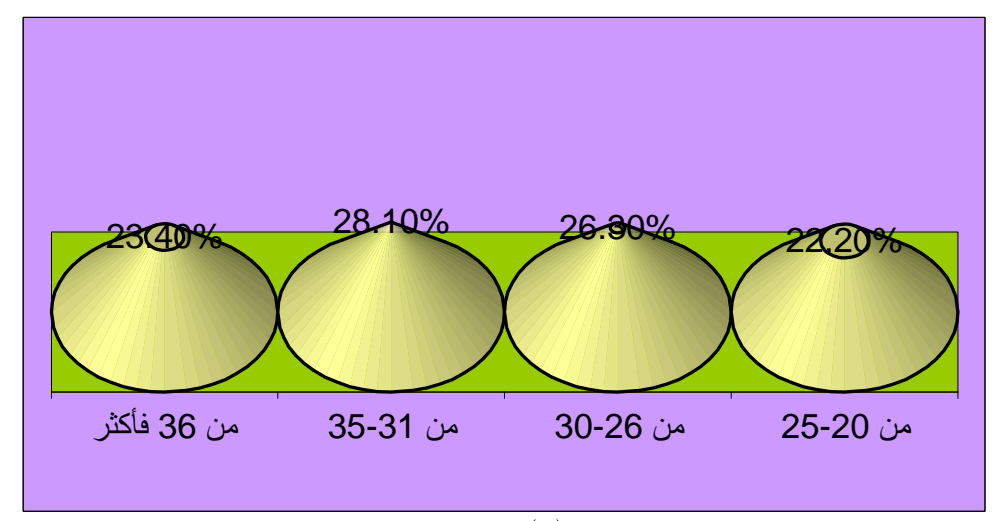

$$
\text { شكل (0) يوضـح السن }
$$

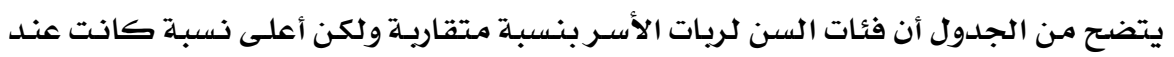

فئة السن من اس- مباسنة 


\section{ثانيًا : ارتباط عناصر العملية الإدارية بمتغيرات الدراسة :}

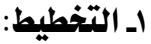

\begin{tabular}{|c|c|c|c|c|c|}
\hline الدעلة & قيمـة (ف) & درجـات الحريـة & متوسط المربعات & مجحموع المربعات & \\
\hline \multirow[t]{3}{*}{$\cdot, \cdot 1$} & \multirow[t]{2}{*}{$\varepsilon \varepsilon, Y$. } & r & $110 \varepsilon$ r,VAV & r\&qrו, & بين المجموعات \\
\hline & & rד & YYI,IVE & EYOVI, YQA & داخل المجموعات \\
\hline & & 177 & & $V V Y \cdot Y, Y 0 \Lambda$ & المجهموع \\
\hline
\end{tabular}

\begin{tabular}{|c|c|c|c|c|c|c|}
\hline الدلالة & قيمة (ت) & درجـات الحرية & العينـة & الانحمراف & المتوسطابي & \\
\hline \multirow{2}{*}{ •., · لصالح } & \multirow{2}{*}{$Y,|\leqslant|-$} & \multirow{2}{*}{ Tr } & rq & $1, \varepsilon Y 0$ & $|\Lambda, 7 Y|$ & اقل مـن متوسط \\
\hline & & & ro & $1 \varepsilon, r r$ & YE, YYQ & متوسط \\
\hline \multirow{2}{*}{ |'••• ثانوي } & \multirow{2}{*}{$\begin{array}{c}- \\
1 \cdot, \varepsilon \leqslant \varepsilon \\
\end{array}$} & \multirow{2}{*}{$\wedge \varepsilon$} & rq & $1, \varepsilon Y 0$ & $|\Lambda, T Y|$ & اقل مـن متوسط \\
\hline & & & ov & $\mathrm{V}, \mathrm{\wedge} \wedge$ & $r r, v \cdot r$ & ثانوي \\
\hline \multirow{2}{*}{ جامعامي فاعلي } & \multirow{2}{*}{$\begin{array}{c}- \\
I \cdot, \varepsilon \nvdash \wedge\end{array}$} & \multirow{2}{*}{ Vr } & $r q$ & $1, \varepsilon Y 0$ & $|\Lambda, T Y|$ & اقل من متوسط \\
\hline & & & $\varepsilon \uparrow$ & YY, E. Y & Tr,IVE & جامعي فأعلى \\
\hline \multirow{2}{*}{ 1 +. ثانوي } & \multirow{2}{*}{$\varepsilon, 1 \wedge 7-$} & \multirow{2}{*}{9.} & ro & $1 \varepsilon, r r$ & YE, YYQ & متوسط \\
\hline & & & ov & $\vee, \neg \wedge \wedge$ & $r, v \cdot r$ & ثانوي \\
\hline \multirow{2}{*}{ جامعـي فاعلى } & \multirow{2}{*}{$\wedge, \vee \wedge \wedge \quad-$} & \multirow{2}{*}{ va } & po & IE, & YE,YYq & متوسط \\
\hline & & & $\varepsilon\urcorner$ & $Y Y, \varepsilon \cdot Y$ & Tr,IVE & جامعي فأ على \\
\hline \multirow{2}{*}{ جامعي فاعلى · لصالح } & \multirow{2}{*}{$\wedge, 9 \vee 1-$} & \multirow{2}{*}{1.1} & ov & $\vee, \Upsilon \wedge \wedge$ & rr,v.r & ثانوي \\
\hline & & & $\varepsilon\rceil$ & YY, E. T & Tr,IVE & جامعي فأعلى \\
\hline
\end{tabular}

$$
\text { جدول ( ه ) يوضح المهنة }
$$

\begin{tabular}{|c|c|c|c|c|c|c|}
\hline الدلالة & قيمـة (ت) & درجـات الحريـة & العينـة & الانحراف المعياري & |المتوسط الحسبابي & \\
\hline \multirow{2}{*}{ غير دال } & \multirow[t]{2}{*}{1,107} & \multirow[t]{2}{*}{170} & 94 & 11,490 & 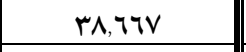 & رية منـزل \\
\hline & & & 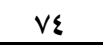 & $Y 0,1 \leqslant 7$ & $r \varepsilon, V V$. & موظفة \\
\hline
\end{tabular}

جدول ( . (1) يوضح عدد الأبناء هِِ الأسرة

\begin{tabular}{|c|c|c|c|c|c|}
\hline الدلالة & قيمـة (ف) & درجـات الحريـة & متوسط المربعات & مجموع المربعات & \\
\hline \multirow[t]{3}{*}{$\cdot, \cdot 1$} & \multirow[t]{2}{*}{$Y \cdot, V Y V$} & r & $V \wedge 7 \cdot, q \cdot r$ & IOVYI,A.T & بين المجموعات \\
\hline & & $17 \varepsilon$ & rVa, Yos & $7 Y 198,090$ & داخل المجموعات \\
\hline & & 177 & & $\operatorname{Vvq19,\varepsilon \cdot 1}$ & المجموع \\
\hline
\end{tabular}




\begin{tabular}{|c|c|c|c|c|c|c|}
\hline الدلالة & قيمـة (ت) & درجات الحرية & العينـة & الانحراف المعياري & المتوسط الحسـابي & \\
\hline \multirow{2}{*}{ | • • • لـ إلى ثلاث واحد } & \multirow{2}{*}{ Y,IYT } & \multirow{2}{*}{ Iry } & 77 & M,TM & צ & من واحد إلى ثلاث | \\
\hline & & & $7 r$ & $r \varepsilon, 0 r$ & 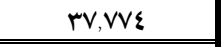 & مـن أربـع إلى سـت \\
\hline \multirow{2}{*}{ | · · • لـصالح واحد } & \multirow{2}{*}{$V, V \leqslant 1$} & \multirow{2}{*}{$1 \cdot r$} & 77 & IN,TrY & $\{0, \wedge \mu r$ & من واحد إلى ثلاث \\
\hline & & & rq & $1 \cdot, 717$ & $r \cdot 07 \varepsilon$ & أكثر من ست \\
\hline \multirow{2}{*}{ | · · · لصالح أربع } & \multirow{2}{*}{$\varepsilon, Y \cdot q$} & \multirow{2}{*}{99} & $\pi$ & $r \xi, 0 r$ & $r V, V V \varepsilon$ & مـن أربـع إلى سـت \\
\hline & & & rq & $1 \cdot, T \Lambda$ & $Y \cdot, 0 T \varepsilon$ & أكثر من ست \\
\hline
\end{tabular}

جدول ( 11 ) يوضـح عدد الخدم ٍِِ الأسـرة

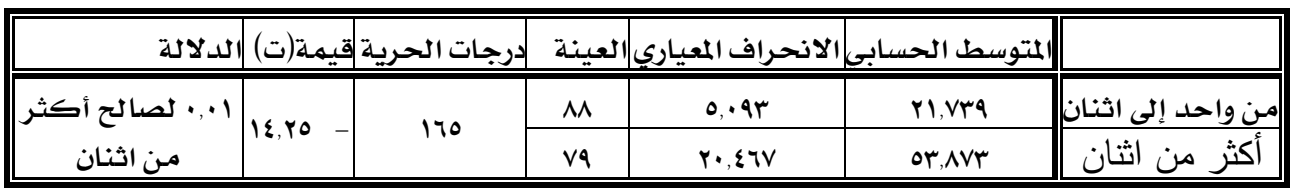

$$
\text { جدول ( rا ) يوضتح السن }
$$

\begin{tabular}{|c|c|c|c|c|c|}
\hline الدلادة & قيمة (ف) & |درجات الحرية & متوسط المربعات & |مججموع المربعات & \\
\hline \multirow{3}{*}{$\cdot, \cdot 1$} & \multirow{2}{*}{ rq,r६१ } & $r$ & $111 \wedge \varepsilon$, rqV & rroor, 19. & بين المجموعات \\
\hline & & 17 r & $Y \wedge \varepsilon, Y \backslash I$ & \&7rrะs,01. & داخل المججموعات \\
\hline & & 177 & & 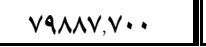 & المجموع \\
\hline
\end{tabular}

\begin{tabular}{|c|c|c|c|c|c|c|}
\hline الدلالة & قيمـة(ت) & درجات الحريـة & العينـة & الانحراف المعياري & |المتوسط الحسـابي & \\
\hline \multirow{2}{*}{ '•., لصـالح من } & \multirow{2}{*}{$\varepsilon, 100-$} & \multirow{2}{*}{ va } & rv & $7, \Lambda \cdot \varepsilon$ & $r \cdot 911$ & من ·ro ro \\
\hline & & & $\varepsilon \varepsilon$ & $1 \Lambda, \cdots 1$ & ד & من דr- م- م \\
\hline \multirow{2}{*}{ ا•, •• لصالح من } & \multirow{2}{*}{$1 \cdot$, YVY - } & \multirow{2}{*}{ Ar } & $r v$ & $7, \Lambda \cdot \varepsilon$ & $r \cdot 911$ & من •ro ro \\
\hline & & & $\varepsilon \vee$ & YI,NTY & $09, Y V T$ & من اس- مب \\
\hline \multirow{2}{*}{ ب ب., · لصأح من } & \multirow{2}{*}{ r,AYr - } & \multirow{2}{*}{ V乏 } & $r v$ & $7, \Lambda \cdot \varepsilon$ & $r \cdot 911$ & من •ro \\
\hline & & & rq & $\Lambda, 0 \wedge \varepsilon$ & rq, IYA & من بس فأكثر \\
\hline \multirow{2}{*}{ ا•., لصـالح من } & \multirow{2}{*}{$0,\{91-$} & \multirow{2}{*}{19} & $\varepsilon \varepsilon$ & $\mid 1, \cdots 1$ & דוז, דוא & من דr- م. \\
\hline & & & $\varepsilon \vee$ & rI,NTr & $09, Y V T$ & من اس- مب \\
\hline \multirow{2}{*}{ ו•., لصالح من } & \multirow{2}{*}{ r.177 } & \multirow{2}{*}{$\wedge 1$} & $\varepsilon \varepsilon$ & $11, \cdots 1$ & דיו, דיז & من דr- م. \\
\hline & & & pq & $\Lambda, 0 \wedge \varepsilon$ & rT, IYA & من بس فأكثر \\
\hline \multirow{2}{*}{ '•., لصـالح من } & \multirow{2}{*}{$1,9 \cdot 9$} & \multirow{2}{*}{$\wedge \varepsilon$} & $\varepsilon V$ & YI,NTY & $09, Y V T$ & من اس- هب \\
\hline & & & rq & $\Lambda, 0 \wedge \varepsilon$ & YT,IYA & من بس فأكثر \\
\hline
\end{tabular}


rـ التنفيذ:

جدول ( سا ) يوضـح المستوى التعليمى

\begin{tabular}{|c|c|c|c|c|c|}
\hline الدلادة & قيمة (ف) & درجـات الحريـة & متوسط المربعات & مجموع المربعات & \\
\hline \multirow{3}{*}{$\cdot, \cdot 1$} & \multirow{2}{*}{$\varepsilon r, \wedge \Lambda$} & $r$ & $r V \leqslant V, 11$. & Arsl,rrq & بين المجموعات \\
\hline & & rד & $7 r, 7.0$ & I.Y.E,Trr & داخل المجموعات \\
\hline & & 177 & & $1 \wedge \leqslant \leqslant 0,90 Y$ & المجموع \\
\hline
\end{tabular}

\begin{tabular}{|c|c|c|c|c|c|c|}
\hline الدلادلة & قيمـة(ت) & درجات الحرية & العينة & الانحراف المعياري & |المتوسط الحسـابي & \\
\hline \multirow{2}{*}{ " م.,· لصالح } & \multirow[t]{2}{*}{ 0,Trr - } & \multirow[t]{2}{*}{ Tr } & rq & $r, 1 \leq \varepsilon$ & $1 \varepsilon, 197$ & اقل من متوسط || \\
\hline & & & ro & r, & 19, roV & متوسط \\
\hline \multirow{2}{*}{ ا•·•• لصانح } & \multirow{2}{*}{ r.,oro - } & \multirow[t]{2}{*}{$\wedge \varepsilon$} & rq & $r, 1 \leq \varepsilon$ & $1 \varepsilon, \wedge 97$ & اقل من متوسط | \\
\hline & & & ov & Y.II. & YY,TI\& & ثانوي \\
\hline \multirow{2}{*}{ جامامعي فاعلي } & \multirow{2}{*}{$r \cdot, \varepsilon \varepsilon \cdot-$} & \multirow[t]{2}{*}{ vr } & rq & $r, 1 \leqslant \varepsilon$ & $1 \varepsilon, \wedge 97$ & اقل من متوسط | \\
\hline & & & $\varepsilon 7$ & $7, r \leqslant 1$ & $\varepsilon \cdot \Lambda \cdot \varepsilon$ & جامعي فأعلى \\
\hline \multirow{2}{*}{ ا'• ثانوي } & \multirow[t]{2}{*}{ Ir,vev - } & \multirow[t]{2}{*}{9.} & ro & r.r.r & 19, roV & متوسط \\
\hline & & & ov & r.11. & YT,TI\& & ثانوي \\
\hline \multirow{2}{*}{ جامـ. • لصالح } & \multirow[t]{2}{*}{ 11,ory - } & \multirow[t]{2}{*}{ Vq } & ro & r.r.r & 19, rov & متوسط \\
\hline & & & $\varepsilon 7$ & $7, r \varepsilon 1$ & $\varepsilon \cdot \Lambda \cdot \varepsilon$ & جامعي فأعلى \\
\hline \multirow{2}{*}{ جامامعي فاعلي } & \multirow[t]{2}{*}{$10,101-$} & \multirow[t]{2}{*}{$1 \cdot 1$} & ov & Y.II. & YY,TI\& & ثانوي \\
\hline & & & $\varepsilon 7$ & $7, r \leqslant 1$ & $\varepsilon \cdot \Lambda \cdot \varepsilon$ & جامعي فأعلى \\
\hline
\end{tabular}

$$
\text { جدول ( \&1) يوضح المهنة }
$$

\begin{tabular}{|c|c|c|c|c|c|c|}
\hline الدلاكة & قيمـة(ت) & درجات الحريـة & العينة & الانحراف المعياري & المتوسط الحسابى & \\
\hline \multirow{2}{*}{ ا•., • لصالح } & \multirow[t]{2}{*}{$1 \varepsilon, \cdot Y \varepsilon$} & \multirow[t]{2}{*}{170} & צ & $\wedge, \leqslant 01$ & ו ו ו & ربـة مـنزل \\
\hline & & & V乏 & $\varepsilon, 0 \wedge \vee$ & 11,819 & موظفة \\
\hline
\end{tabular}

$$
\text { جدول ( } 10 \text { ) يوضح عدد الأبناء يِّالأسـرة }
$$

\begin{tabular}{|c|c|c|c|c|c|}
\hline الدلالة & قيمة (ف) & درجـات الحـرية & متوسط المربعات & مججموع المربعات & \\
\hline \multirow{2}{*}{$\cdot, \cdot 1$} & \multirow{2}{*}{$0 \leqslant, 7 \leqslant 9$} & r & rvro,011 & $V \Sigma V I, \cdot r Y$ & بين المجموعات \\
\hline & & $17 \varepsilon$ & TA, roo & $\| Y \mid \cdot, r Y \varepsilon$ & داخل المجمـوعات \\
\hline & & 177 & & |NTAI,YะT & المجهوع \\
\hline
\end{tabular}




\begin{tabular}{|c|c|c|c|c|c|c|}
\hline الدلادة & قيمـة(ت) & درجات الحرية & العينة & الانحراف المعياري & المتوسط الحسـابي & \\
\hline \multirow{2}{*}{ 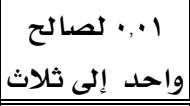 } & \multirow{2}{*}{$1 Y, \cdot V 7$} & \multirow{2}{*}{ IrT } & 77 & $\vee, 90 \wedge$ & m,ars & من واحد إلى ثلاث| \\
\hline & & & Tr & $r, \varepsilon \wedge \vee$ & rr,TII & مـن أربـع إلى ست \\
\hline \multirow{2}{*}{ 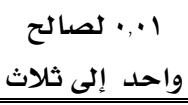 } & \multirow{2}{*}{$17, r \leqslant V$} & \multirow{2}{*}{$1 \cdot r$} & 77 & $V, 901$ & M., & من واحد إلى ثلاث| \\
\hline & & & rq & $r .90$ & $10, Y A Y$ & أكثر من ست \\
\hline \multirow{2}{*}{ أ. أربع إلى سـالح } & \multirow{2}{*}{ IY,YVY } & \multirow{2}{*}{99} & Tr & $\boldsymbol{r}, \boldsymbol{\wedge} \wedge$ & Yr,TYI & مـن أربـع إلى سـت \\
\hline & & & rq & $r .90$ & 10,YAY & أكثر من ست \\
\hline
\end{tabular}

جدول ( 17 ) يوضـح عدد الخدم ِِ الأسـرة

\begin{tabular}{|c|c|c|c|c|c|c|}
\hline الدلالة & قيمة (ت) & درجـات الحرية & |العينة & الانحراف المعياري & |المتوسط الحسابي & \\
\hline \multirow{2}{*}{ أ., أكثر من اثنان } & \multirow{2}{*}{$\wedge, \varepsilon \varepsilon \cdot \quad-$} & \multirow{2}{*}{170} & $\wedge \wedge$ & $V, V \vee 1$ & rI, OVq & من واحد إلى اثنـان \\
\hline & & & va & 9,01 & rY,QYร & أكثر مـن اثنان \\
\hline
\end{tabular}

جدول ( IV ) يوضتح السن

\begin{tabular}{|c|c|c|c|c|c|}
\hline الدلالة & قيمة (ف) & درجـات الحـريـة & متوسط المربعات & مجهموع المربعات & \\
\hline \multirow[t]{3}{*}{$\cdot, \cdot 1$} & \multirow[t]{2}{*}{$0 ., 119$} & $r$ & YATY, $\varepsilon \varepsilon r$ & NONV,rYA & بين المجموعات \\
\hline & & r & OT,YYT & $91 \wedge 1,1 \wedge \mathrm{V}$ & داخل المجموعات \\
\hline & & 177 & & $1 V V 71,010$ & المجموع \\
\hline
\end{tabular}

\begin{tabular}{|c|c|c|c|c|c|c|}
\hline الدلالة & |قيمـة(ت) & درجـات الحريـة & |العينـة | & الانحراف المعياري| & |المتوسط الحسـابي & \\
\hline \multirow{2}{*}{ |'• • لهـالح } & \multirow{2}{*}{$7, \cdot r \varepsilon-$} & \multirow{2}{*}{ Vq } & m & r,roz & Yr,ITr & مـ ro ro r \\
\hline & & & $\varepsilon \varepsilon$ & A.rYI & $r \cdot, 900$ & 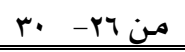 \\
\hline \multirow{2}{*}{ |+. • لصن اسلح } & \multirow{2}{*}{$V, \leqslant M V-$} & \multirow{2}{*}{$\Lambda r$} & m & r,ros & rY,ITr & من ·ro \\
\hline & & & $\varepsilon V$ & $1 \cdot, Y Y 1$ & 20,191 & من اس- 0ر \\
\hline \multirow{2}{*}{ 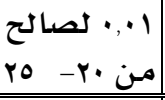 } & \multirow{2}{*}{$0,1 \leqslant 9$} & \multirow{2}{*}{ 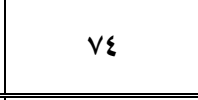 } & m & r,roz & rY,ITr & من ·ro \\
\hline & & & rq & $0,11 Y$ & IV, ro & مـن بس فأكثر \\
\hline \multirow{2}{*}{ ه•. • لصنالح } & \multirow{2}{*}{ Y.17. - } & \multirow{2}{*}{19} & $\varepsilon \varepsilon$ & A.rYI & $r \cdot 900$ & 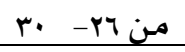 \\
\hline & & & $\varepsilon \vee$ & $1 \cdot, Y Y I$ & 20,191 & من اس- 0س \\
\hline \multirow{2}{*}{ |'• • لصنالح } & \multirow{2}{*}{$9 \cdot \div 87$} & \multirow{2}{*}{$\Lambda 1$} & $\varepsilon \varepsilon$ & A.rYI & $r \cdot, 900$ & من بr- . \\
\hline & & & rq & $0,11 Y$ & IV, Y YO & من بس فأكثر \\
\hline \multirow{2}{*}{ 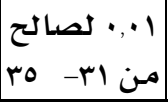 } & \multirow{2}{*}{$1 \cdot, \cdot 9 \varepsilon$} & \multirow{2}{*}{$\wedge \varepsilon$} & $\varepsilon \vee$ & 1.,YYI & $r 0,191$ & من اس- هر \\
\hline & & & rq & $0,11 Y$ & $I V, \cdot Y_{0}$ & مـن جس فأكثر \\
\hline
\end{tabular}




\begin{tabular}{|c|c|c|c|c|c|}
\hline الدلالة & قيمة (ف) & درجـات الحـريـة & متوسط المريعات & مجمهوع المربعات & \\
\hline \multirow{2}{*}{$\cdot, \cdot 1$} & \multirow{2}{*}{$0 \&, \cdot 17$} & $r$ & $\varepsilon Q \vee \varepsilon, Y \circ\}$ & |\&QYr,VT| & بين المجموعات \\
\hline & & מד1 & $9 r, \cdot 19$ & $10 \cdot 1 \cdot 0 \cdot 1$ & داخل المجموعات \\
\hline & & 177 & & r৭৭rr, Yרq & المجموع \\
\hline
\end{tabular}

\begin{tabular}{|c|c|c|c|c|c|c|}
\hline الدلادلة & قيمةة(ت) & درجات الحريـة| & العينة & |الانحراف المعياري & |المتوسط الحسـابي & \\
\hline \multirow{2}{*}{ اهץ, • غير دال } & \multirow{2}{*}{$1,70-$} & \multirow{2}{*}{ Tr } & rq & 9,1 ro & 19,var & |اقل من متوسط|| \\
\hline & & & ro & $7, \wedge \cdot$ & YI,AYq & متوسط \\
\hline \multirow{2}{*}{ | •., · لصالح ثانوي| } & \multirow{2}{*}{$\mathrm{V}, \mathrm{N} 19-$} & \multirow{2}{*}{$\wedge \varepsilon$} & rq & 9.1 ro & 19,var & |اقل مـن متوسط| \\
\hline & & & ov & $0,11 \mathrm{r}$ & rI,V^9 & ثانوي \\
\hline \multirow{2}{*}{ جامعـي فاعلي } & \multirow{2}{*}{$1 \cdot, 11 \cdot-$} & \multirow{2}{*}{$v r$} & rq & 9,1 ro & 19,var & |اقل من متوسط|| \\
\hline & & & $\varepsilon 7$ & $\mid Y, Y O 1$ & $\{7,0 \leqslant\}$ & جامعي فأعلى \\
\hline \multirow{2}{*}{ | •., · لصالح ثانوي| } & \multirow{2}{*}{$\Lambda, \varepsilon r V-$} & \multirow{2}{*}{9.} & ro & $7 \cdot \wedge \cdot$ & rI,Arq & متوسط \\
\hline & & & ov & $0,11 \mathrm{r}$ & rI,V^q & ثانوي \\
\hline \multirow{2}{*}{ جامعـي فاعلي } & \multirow{2}{*}{$1 \cdot, 9 \leqslant Y-$} & \multirow{2}{*}{ va } & ro & $7 \cdot \wedge \cdot$ & rI,AYq & متوسط \\
\hline & & & $\sum 7$ & $\mid Y, Y O 1$ & $\{7,0 \leqslant\}$ & جامعي فأعلى \\
\hline \multirow{2}{*}{ جامعي فاعلي } & \multirow{2}{*}{ A,Yor - } & \multirow{2}{*}{$1 \cdot 1$} & ov & $0,11 Y$ & rI,V^9 & ثانوي \\
\hline & & & $\varepsilon$ & $|r, Y O|$ & $\{7,0 \leqslant \varepsilon$ & عي فأعلى \\
\hline
\end{tabular}

$$
\text { جدول ( } 19 \text { ) يوضـح المهنة }
$$

\begin{tabular}{|c|c|c|c|c|c|c|}
\hline الدلاكلة & قيمـة(ت) & درجـات الحـريـة & العينة & الانحـراف المعياري & المتوسط الحسسابي & \\
\hline \multirow{2}{*}{ غير دال } & \multirow[t]{2}{*}{ 1, roq - } & \multirow[t]{2}{*}{170} & ra & 11,919 & $r \cdot$ orv & ربـة منـزل \\
\hline & & & V乏 & $1 \varepsilon, V \wedge \Lambda$ & סקון & موظفة \\
\hline
\end{tabular}

جدول ( • ب يوضح عدد الأبناء يِّ الأسرة

\begin{tabular}{|c|c|c|c|c|c|}
\hline الدلالة & قيمـة (ف) & درجـات الحريـة & متوسط المربعات & مجسموع المربعات & \\
\hline \multirow{3}{*}{$\cdot, \cdot 1$} & \multirow{2}{*}{ דY } & $r$ & $70 \leqslant Y, \wedge \wedge \Lambda$ & $1 r \cdot \wedge 0, v V 0$ & بين المجمهوعات \\
\hline & & $17 \varepsilon$ & IIT,r $\varepsilon V$ & $19 \cdot 1 \cdot, 941$ & داخل المججموعات \\
\hline & & 177 & & rYITY,V.Y & المجموع \\
\hline
\end{tabular}




\begin{tabular}{|c|c|c|c|c|c|c|}
\hline الدلادة & قيمـة(ت) & درجات الحرية & |العينة | & الانحراف المعياري & |المتوسط الحسسابي & \\
\hline \multirow{2}{*}{ וY • • غير دال } & \multirow{2}{*}{$1,1.0$} & \multirow{2}{*}{ IYT } & 77 & $1 \cdot, \Lambda 1 r$ & 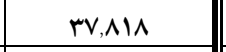 & من واحد إلى ثلاث \\
\hline & & & Tr & $1 \cdot, 9 \varepsilon$. & ro,79\& & من أربـع إلى ست \\
\hline \multirow{2}{*}{ إ... · لصالح } & \multirow{2}{*}{$1 r \cdot \wedge 0$} & \multirow{2}{*}{$1 \cdot r$} & 77 & $1 \cdot, \Lambda 1 r$ & 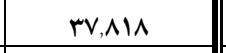 & من واحد إلى ثلاث \\
\hline & & & rq & 1,179 & 1\&,9Yr & أكثر من ست \\
\hline \multirow{2}{*}{ أ.ر. • لصـع إلى ستح } & \multirow{2}{*}{ II,VYA } & \multirow{2}{*}{99} & $\pi$ & $1 \cdot, 9 \varepsilon$. & ro,79q & من أربع إلى ست \\
\hline & & & pq & 1,179 & 1\&,9Yr & أكثر من ست \\
\hline
\end{tabular}

جدول ( ا r) يوضح عدد الخدم ِِِ الأسرة

\begin{tabular}{|c|c|c|c|c|c|c|}
\hline الدلادة & قيمـة(ت) & درجات الحرية & |العينة & الانحراف المعياري & |المتوسط الحسـابي & \\
\hline \multirow{2}{*}{ | أ., · لصثالح مـن اثنان } & \multirow{2}{*}{$10, Y \cdot 0-$} & \multirow{2}{*}{170} & $\Lambda$ & T,YAV & $M Y, \cdot T \Lambda$ & من واحد إلى اثنان \\
\hline & & & va & $1 \cdot, T \leqslant Y$ & \&r,rqr & أكثر من اثنان \\
\hline
\end{tabular}

$$
\text { جدول ( r ) يوضتح السن }
$$

\begin{tabular}{|c|c|c|c|c|c|}
\hline الدلادة & قيمـة (ف) & درجات الحرية & متوسط المربعات & مـجموع المربعات & \\
\hline \multirow[t]{3}{*}{$\cdot, \cdot 1$} & \multirow[t]{2}{*}{$0 V, 797$} & r r r r r & OYVO,OYY & IONYY,OVV & بين المجهوعات \\
\hline & & rit & q1, \&rV & $|\varepsilon q \cdot \varepsilon, Y 7|$ & داخل المجموعات \\
\hline & & 177 & & r.v & المجهوع \\
\hline
\end{tabular}

\begin{tabular}{|c|c|c|c|c|c|c|}
\hline الدلالة & قيمـة (ت) & |درجـات الحرية | & العينة & |الانحراف المعياري & المتوسط الحسـابي & \\
\hline \multirow{2}{*}{ 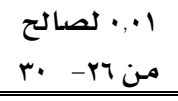 } & \multirow{2}{*}{ Q,Arr - } & \multirow{2}{*}{ va } & rv & $\wedge, 94$. & $1 V, 09 \varepsilon$ & من •ro \\
\hline & & & $\varepsilon \varepsilon$ & T,rYV & $r \leqslant, r \mid \Lambda$ & 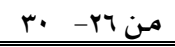 \\
\hline \multirow{2}{*}{ 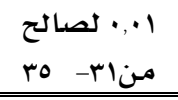 } & \multirow{2}{*}{ Ir,IrV - } & \multirow{2}{*}{ Ar } & rv & $\Lambda, 9 r$. & $1 V, 09 \varepsilon$ & من ·ro ro \\
\hline & & & $\varepsilon V$ & $11,0 \mathrm{VV}$ & $\{0,097$ & من اس- هب \\
\hline \multirow{2}{*}{ من '• • لصأح } & \multirow{2}{*}{$0, V Y-$} & \multirow{2}{*}{$V \varepsilon$} & rV & $\Lambda, 94$. & $1 V, 09 \varepsilon$ & ro ro r r r \\
\hline & & & mq & $r, 100$ & $r o, r \cdot \Lambda$ & من بس فأكثر \\
\hline \multirow{2}{*}{ "•. • لصنالح } & \multirow{2}{*}{$0, \times 11-$} & \multirow{2}{*}{19} & $\varepsilon \varepsilon$ & T,YYV & $r \varepsilon, r \mid \Lambda$ & من צr- . \\
\hline & & & $\varepsilon V$ & $11,0 \mathrm{VV}$ & $\{0,097$ & من اس- هب \\
\hline \multirow{2}{*}{ 1•, • لصالح } & \multirow{2}{*}{$\Lambda \cdot \bullet \varepsilon \vee$} & \multirow{2}{*}{$\wedge 1$} & $\varepsilon \varepsilon$ & ר,YYV & r\&,rin & من דr- . \\
\hline & & & rq & $r, 100$ & $r o, r \cdot \Lambda$ & مـن بس فأكثر \\
\hline \multirow{2}{*}{ م-., • لصالح } & \multirow{2}{*}{$1 \cdot, 71 Y$} & \multirow{2}{*}{$\wedge \varepsilon$} & $\varepsilon V$ & $11,0 \mathrm{VV}$ & $\{0,097$ & من اس- هر \\
\hline & & & rq & $r, 100$ & $r 0, r \cdot \Lambda$ & مـن بس فأكثر \\
\hline
\end{tabular}




\begin{tabular}{|c|c|c|c|c|c|}
\hline الدلادة & قيمـة (ف) & درجات الحريـة & متتوسط المربعات & مجسموع المربعات & \\
\hline \multirow[t]{3}{*}{$\cdot, \cdot 1$} & \multirow[t]{2}{*}{$09, \Lambda \cdot r$} & $r$ & $19 \varepsilon \cdot \wedge, r \wedge \varepsilon$ & OAYYO, 101 & بين المجمهوعات \\
\hline & & $17 \pi$ & rys,org & OY^৭9,V৭० & داخل المجميموعات \\
\hline & & 177 & & $1111 \times \varepsilon, 9 \varepsilon 7$ & المجموع \\
\hline
\end{tabular}

\begin{tabular}{|c|c|c|c|c|c|c|}
\hline الدلالة & قيمـة(ت) & |درجات الحـريـة & العينة & الانحراف المعياري & المتوسط الحسـابى & \\
\hline \multirow{2}{*}{ '•., متوسطالح } & \multirow{2}{*}{ r.ONI - } & \multirow{2}{*}{$7 r$} & rq & r,YuA & $r r, \cdot r \varepsilon$ & اقل من متوسط \\
\hline & & & ro & $1 \varepsilon, r V r$ & $r Y, V \varepsilon r$ & متوسط \\
\hline \multirow{2}{*}{ 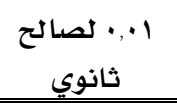 } & \multirow{2}{*}{ Ir,IVY - } & \multirow{2}{*}{$\wedge \varepsilon$} & rq & r, rus & $r r, r \varepsilon$ & اقل من متوسط \\
\hline & & & ov & $1 \varepsilon, 1 \cdot V$ & $00, \varepsilon r q$ & ثانوي \\
\hline \multirow{2}{*}{ جامعي فأعلى } & \multirow{2}{*}{$19 \cdot v \cdot-$} & \multirow{2}{*}{ Vr } & rq & r,YMA & & اقل من متوسط \\
\hline & & & $\varepsilon 7$ & $10, \Lambda 1 Y$ & Vq,91r & جامعي فأعلى \\
\hline \multirow{2}{*}{ 1 +. • لصانوي } & \multirow{2}{*}{$V, \S 09-$} & \multirow{2}{*}{9.} & ro & $1 \varepsilon, r V r$ & $r Y, V \varepsilon r$ & متوسط \\
\hline & & & ov & $1 \leqslant, 1 \cdot V$ & $00, \varepsilon r q$ & ثانوي \\
\hline \multirow{2}{*}{ جامعي فأعلى } & \multirow{2}{*}{ Ir,人TE - } & \multirow{2}{*}{ va } & ro & $1 \varepsilon, Y V r$ & rY,VEr & متوسط \\
\hline & & & $\varepsilon 7$ & $10, \Lambda 1 Y$ & va,91r & جامعي فأعلى \\
\hline \multirow{2}{*}{ جامعي فأعلى } & \multirow{2}{*}{ A.rar - } & \multirow{2}{*}{$1 \cdot 1$} & ov & $1 \varepsilon, 1 \cdot V$ & $00, \varepsilon r q$ & ثانوي \\
\hline & & & $\varepsilon 7$ & $10, \Lambda \mid Y$ & va,91r & جامعي فأعلى \\
\hline
\end{tabular}

$$
\text { جدول (r乏) يوضح المهنة }
$$

\begin{tabular}{|c|c|c|c|c|c|c|}
\hline الدلالة & قيمة (ت) & درجات الحريـة & العينة & الانحراف المعياري & المتوسط الحسـابي & \\
\hline \multirow{2}{*}{ ا•., • لصالح } & \multirow{2}{*}{$r V, r V r-$} & \multirow{2}{*}{170} & 94 & $1 \cdot, 709$ & $r 1,799$ & ربـة مـنزل \\
\hline & & & $V \varepsilon$ & $1 \cdot, 7 \cdot V$ & $V V, \cdot 0 \varepsilon$ & موظفة \\
\hline
\end{tabular}

جدول ( ro ) يوضتح عدد الأبناء يِّ الأسرة

\begin{tabular}{|c|c|c|c|c|c|}
\hline الدلالدة & قيمة (ف) & درجـات الحـرية & متوسط المربعات & مـجموع المربعات & \\
\hline \multirow{2}{*}{$\cdot, \cdot 1$} & \multirow{2}{*}{ צrד, } & r & $1077 V .49$ & & بين المجمهوعات \\
\hline & & 178 & $\varepsilon r q, 770$ & $V Y I \cdot 0, \cdots$ & داخل المجموعات \\
\hline & & 177 & & $1 \cdot r \leqslant r q, v \wedge$ & المجموع \\
\hline
\end{tabular}




\begin{tabular}{|c|c|c|c|c|c|c|}
\hline الدلاكة & قيمـة(ت) & |درجات الحريـة & العينة & الانحراف المعياري & |المتوسط الحسـابي & \\
\hline \multirow{2}{*}{ 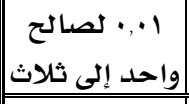 } & \multirow{2}{*}{$7,0\} \wedge$} & \multirow{2}{*}{ IYY } & 77 & ro..09 & רu,ryE & من واحد إلى ثلاث \\
\hline & & & Tr & $10,1 \mathrm{M}$ & $\varepsilon r, q \cdot r$ & مـن أربـع إلى ست \\
\hline \multirow{2}{*}{ | إحد. • لصالح إلى ثلاث } & \multirow{2}{*}{$7, V 7 \varepsilon$} & \multirow{2}{*}{$1 \cdot r$} & 77 & $r 0 . .09$ & Th,rTE & من واحد إلى ثلاث \\
\hline & & & rq & $r \cdot, \varepsilon \leqslant \Lambda$ & $r, r \cdot v$ & أكثر من ست \\
\hline \multirow{2}{*}{ ه•. أربع إلى سالح } & \multirow{2}{*}{$r, 91$} & \multirow{2}{*}{99} & $\pi$ & $10,1 \mathrm{M} \Lambda$ & $\varepsilon r, q \cdot r$ & مـن أربـع إلى ست \\
\hline & & & rq & $Y \cdot, \varepsilon \leqslant \Lambda$ & $r, r \cdot v$ & أكثر من ست \\
\hline
\end{tabular}

جدول ( جr) يوضح عدد الخدم يِّ الأسرة

\begin{tabular}{|c|c|c|c|c|c|c|}
\hline الدلالة & قيمـة(ت) & درجـات الحرية & العينة & الانحراف المعياري & المتوسط الحسـابي & \\
\hline \multirow{2}{*}{ أ أ, • لصـالح } & \multirow{2}{*}{$r V, 999$} & \multirow{2}{*}{170} & $\Lambda$ & Q,YTV & rוז,re & مـن واحد إلى اثنـان|| \\
\hline & & & va & $11,7 \cdot 9$ & Vo,TVI & أكثر من اثنان \\
\hline
\end{tabular}

$$
\text { جدول ( rv ) يوضتح السن }
$$

\begin{tabular}{|c|c|c|c|c|c|}
\hline الدلادة & قيمة (ف) & درجات الحريـة & متوسط المربعات & مجموع المربعات & \\
\hline \multirow{3}{*}{$\cdot \cdot \cdot 1$} & \multirow{2}{*}{$\varepsilon 7, \cdot 19$} & $r$ & $17 . .9, v 74$ & $\varepsilon \wedge \cdot Y q, Y \wedge q$ & بين المجموعات \\
\hline & & 174 & r\&V,^৭r & $0 T V \cdot Y, \varepsilon \wedge \varepsilon$ & داخل المجمهوعات \\
\hline & & 177 & & $1 . \varepsilon V r o, V V r$ & المجموع \\
\hline
\end{tabular}

\begin{tabular}{|c|c|c|c|c|c|c|}
\hline الدلالة & قيمةت(ت) & درجات الحرية & العينـة & الانحراف المعياري & المتوسط الحسـابى & \\
\hline \multirow{2}{*}{ 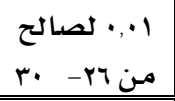 } & \multirow{2}{*}{$1 \cdot, \leqslant \leqslant 0 \quad-$} & \multirow{2}{*}{ va } & $r v$ & $1 ., 091$ & YV,rYY & من ·ro ro r \\
\hline & & & $\varepsilon \varepsilon$ & $11,17$. & $\pi r, V \cdot 0$ & من بr- م \\
\hline \multirow{2}{*}{ من إب- • لصالح } & \multirow{2}{*}{$11, \wedge \mathrm{VV}-$} & \multirow{2}{*}{ Ar } & rv & 1.091 & YV,rYE & من ·ro \\
\hline & & & $\varepsilon \vee$ & $r \cdot, \varepsilon \mid r$ & VI, rres & من اس- هب \\
\hline \multirow{2}{*}{ أ. من بس فأكثر } & \multirow{2}{*}{ r,YTT - } & \multirow{2}{*}{$\mathrm{V} \varepsilon$} & $r v$ & $1 \cdot .091$ & YV,rYE & من ·r \\
\hline & & & rq & $19, \mathrm{Vr} 1$ & rQ, YAY & من بس فأكثر \\
\hline \multirow{2}{*}{ ه., • لصالح } & \multirow{2}{*}{$Y, 1+1=$} & \multirow{2}{*}{19} & $\varepsilon \varepsilon$ & $11,17$. & $T r, V \cdot 0$ & r. من דr- r. \\
\hline & & & $\varepsilon \vee$ & $r \cdot, \varepsilon \mid r$ & VI, rre & من اس- مب \\
\hline \multirow{2}{*}{ "•. • لصالح } & \multirow{2}{*}{ ו ו } & \multirow{2}{*}{$\wedge 1$} & $\varepsilon \varepsilon$ & $11,17$. & $T r, V \cdot 0$ & r. من بr- r. \\
\hline & & & rq & $19, \mathrm{VH}$ & rq, YAY & من بس فأكثر \\
\hline \multirow{2}{*}{ '••• لصنالح } & \multirow{2}{*}{ ר,rre } & \multirow{2}{*}{$\wedge \varepsilon$} & $\varepsilon \vee$ & $r \cdot, \varepsilon \mid r$ & VI, rres & من اس- هب \\
\hline & & & rq & $19, V K 1$ & rq, rAr & من بس فأكثر \\
\hline
\end{tabular}


جدول ( ) يوضح مصفوفة ارتباط بين عناصر العملية الإدارية و متغيرات الدراسة

\begin{tabular}{|c|c|c|c|c|c|}
\hline الاستمارة كلها & إدارة الوقت & التقييم & التنفيذ & التخطيط & مصفوفة ارتباط \\
\hline$* * \cdot \wedge 9 r$ & $* * \cdot \wedge \vee 1$ & $* * \cdot \operatorname{var}$ & $* * \cdot \wedge \mid r$ & $* *, \wedge \wedge 0$ & المستوى التعليهي \\
\hline$* * .911$ & $* *, \wedge T 7$ & $\%$, voo & $\$ \% 910$ & 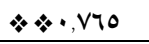 & المهنة \\
\hline$* *, \wedge I r$ & $* *, \vee 99$ & $* * \cdot 9 r r$ & $* \bullet \wedge \wedge \wedge$ & $* \bullet, \wedge T 1$ & عدد الأبناء يِّالأسـرة \\
\hline$* *, 94 \varepsilon$ & $* \bullet, \wedge 97$ & $* * \cdot \mathrm{VVT}$ & 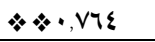 & ד & عدد الخدم ٍِِ الأسرة \\
\hline$* * \cdot$ AY & $* *$, ^or & $* * \cdot 9 \cdot 1$ & $* *, \wedge \leq \varepsilon$ & *.,vAr & السن \\
\hline
\end{tabular}

\section{نتائج البحث :}

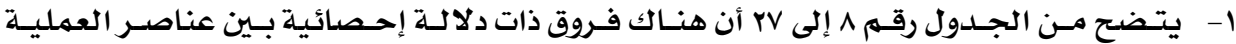

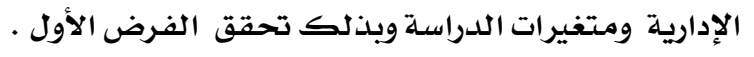

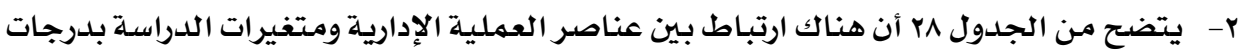

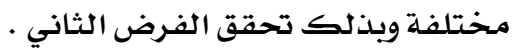

التوصيات :

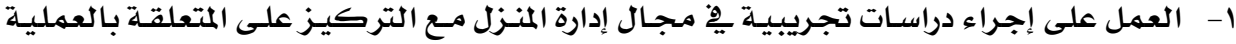

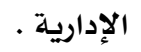

r- ت توعية الأسرة من خلال البر امج المخصصة بأهميلة إدارة موارد الأسرة .

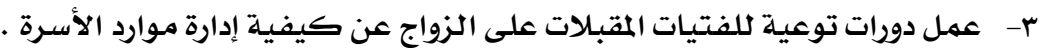

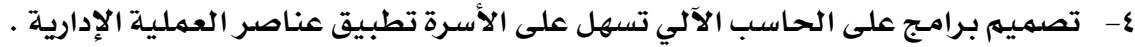


1- توفيق ، احمد جميل (9191م) :" إدارة الأعمال مدخل وظيفي " دار النهضدة ، بيروت .

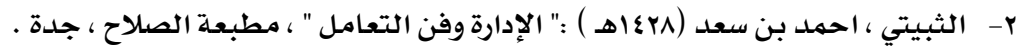

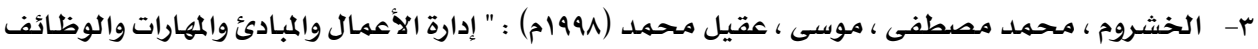

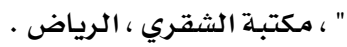

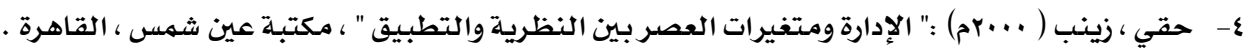

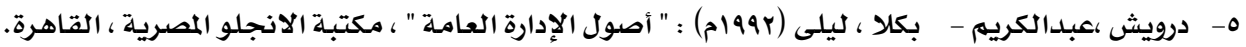

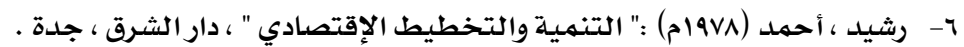

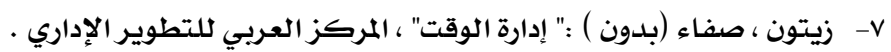

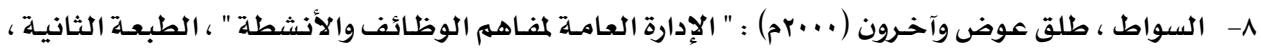
دار حافظ ، جدة .

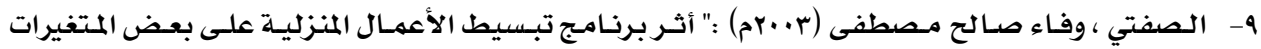

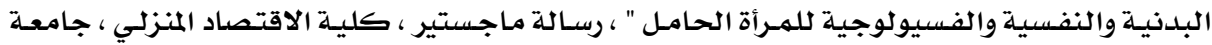

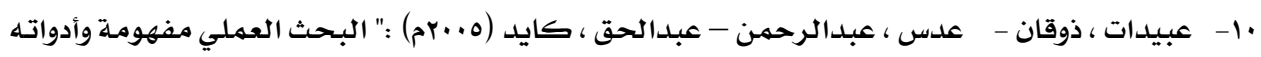

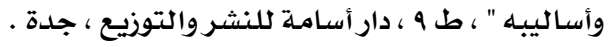

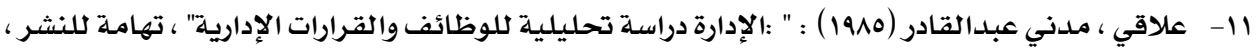
جدة .

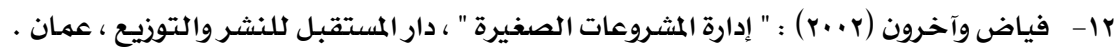

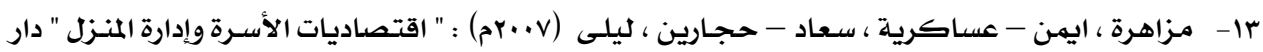

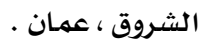

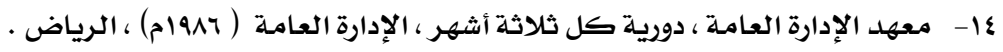

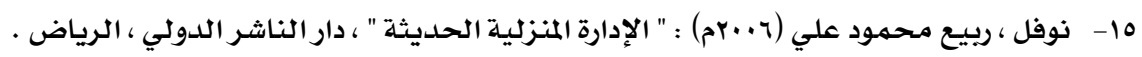

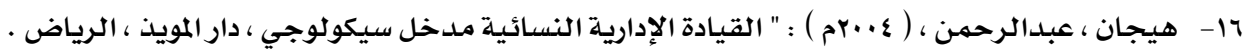
17-AHAND BOOK OF MANAGEMEN Techniques(2002) janin , Michael Armstrong Book stor. Riyad.

18-G. Starling Managing the bubic sector ( Homewood: The Dorsey press, 1982) . P.302.

19-H.Koontz and C.o Donnel, Principles of Management , (N.Y.McGraw Hill Book Co. (1979) P.710. 
